\title{
Geoestatística aplicada na determinação da compactação do solo no cultivo da cana-de-açúcar
}

\author{
Indiamara Marasca ${ }^{1}$, Joanina Gladenucci ${ }^{2}$, Emanuel Rangel Spadim², Rodrigo Domingues \\ Barbosa $^{3}$, Kleber Pereira Lanças ${ }^{2}$ \\ ${ }^{1}$ Universidade de Rio Verde Goiás - UniRV, Campus de Rio Verde, Rio Verde, Goiás, Brasil. E-mail: marasca_7@ hotmail.com. \\ ${ }^{2}$ Universidade Estadual Paulista Júlio de Mesquita Filho-FCA/UNESP, Campus de Botucatu, Botucatu, São Paulo, Brasil. E-mail: \\ jogladenucci@gmail.com, spadim@gmail.com, kplancas@ fca.unesp.br \\ ${ }^{3}$ Faculdades Integradas de Bauru, Bauru, São Paulo, Brasil. E-mail: tuvira-rdb@bol.com
}

Recebido: 06/02/2017; Aceito: 14/06/2017.

\section{RESUMO}

As ferramentas de agricultura de precisão diversificam-se cada vez mais para facilitar a tomada de decisão no campo com o intuito de aumentar a produtividade das culturas para alimentar uma população crescente. A responsabilidade dos profissionais das áreas agronômicas deve ser embasada em estudo e domínio de novas tecnologias. Com esta pesquisa objetivou-se demonstrar a técnica de interpolação de dados para confeccionar mapas de solos utilizando software Gamma Desing e as técnicas da geoestatística. A resistência à penetração do solo foi obtida até a profundidade de $0,45 \mathrm{~m}$. Para confecção dos mapas dos transeptos para os tratamentos foi utilizado o software Gamma Desing. Os dados para a camada de 0,30 a $0,45 \mathrm{~m}$ no preparo convencional foi o único que não apresentou dependência espacial. $\mathrm{O}$ uso da geoestatística como ferramenta na agricultura teve resposta satisfatória e o programa Gamma Desing mostrou-se confiável na interpolação dos mapas para a variável de compactação do solo.

Palavras-chave: Índice de cone, variabilidade espacial, preparo profundo.

\section{Geostatistics applied in soil compaction determination in sugar cane cropping}

\begin{abstract}
The tools of precision agriculture increasingly diversify to facilitate decision making in the field in order to increase crop yields to feed a population that only grows. The responsibility of professional in agronomic areas must be grounded in the study and mastery of new technologies. The objective of this work was to demonstrate the technique of data interpolation to make soil maps using Gamma Desing software and the study of geostatistics. The soil penetration resistance was obtained to a depth of $0.45 \mathrm{~m}$. Gamma Desing software was used for the transepts maps preparation for treatments. Data showed that the layer from 0.30 to $0.45 \mathrm{~m}$ in conventional tillage was the only one which showed no spatial dependence. The use of geostatistics as a tool in agriculture had satisfactory response and the Gamma Desing program was reliable in maps interpolation for the soil compaction variable.
\end{abstract}

Key words: Cone index, spatial variability, deep preparation. 


\section{Introdução}

A cana-de-açúcar (Saccharum officinarum L.) é responsável por duas principais "commodities" brasileiras - açúcar e etanol. A produção de cana-de-açúcar para a safra 2015/16 é de 665,6 milhões de toneladas. O crescimento foi de $4,9 \%$ em relação à safra anterior. A área cultiva foi de 8.654,2 mil hectares; redução de 3,9\% comparada com a safra 2014/15. A produção de açúcar atingiu 33,5 milhões de toneladas; 5,8\% inferior à safra 2014/15. A produção de etanol total foi de 30,5 bilhões de litros; aumento de $6,3 \%$. A produção de etanol anidro teve redução de 4,4\%, aprovação de cerca de 11,2 bilhões de litros. Para a produção de etanol hidratado o total foi de 19,3 bilhões de litros; aumento de $13,7 \%$ ou 2,3 bilhões de litros (CONAB, 2016).

Em razão de suas características de clima e solo, o Brasil é o país com melhores condições para gerar e produzir fontes energéticas alternativas ao petróleo como o álcool, derivado da cana-de-açúcar (etanol), figurando como alternativa que mais atende às exigências das economias mundiais por ser renovável e menos poluente (SEGATO et al., 2006).

Com a adoção da colheita mecanizada, no sistema de manejo da cana-de-açúcar, surge a preocupação com a compactação do solo e seus efeitos sobre os atributos físicos do solo. A desestruturação física do solo afeta sua qualidade química e biológica, com reflexos na produtividade da cultura e nos aspectos ambientais da produção (PACHECO; CANTALICE, 2011; VASCONCELOS et al., 2012).

Do plantio à colheita, o cultivo da cana é um processo altamente mecanizado (PAULINO et al, 2004) e a intensidade do tráfego de máquinas causa degradação física do solo criando obstáculos para o crescimento das raízes (CARRARA et al., 2007).

A geoestatística como ciência consagrada e integrante da estatística aplicada trata de problemas referentes às variáveis regionalizadas as quais têm um comportamento espacial mostrando características intermediárias entre as variáveis verdadeiramente aleatórias e as totalmente determinísticas. No estudo do comportamento das variáveis regionalizadas duas são as ferramentas fundamentais dos métodos geoestatísticos: o semivariograma e a krigagem (LANDIM, 2006).

O objetivo deste trabalho foi demonstrar a técnica de interpolação de dados para confeccionar mapas de solos utilizando software Gamma Desing e os pressupostos da geoestatística.

\section{Material e Métodos}

O trabalho foi realizado na empresa PHD Cana, no município de Lençóis Paulista - SP (22\%40’30" Sul e 48 53'30" W), parceira da Usina Zilor. O equipamento utilizado de preparo intensivo, profundo e canteirizado do solo possibilitou a realização de cinco operações ou funções ao mesmo tempo, sendo desta forma denominado vulgarmente por "Penta". Constituído por componentes tais como: uma haste subsoladora, um mecanismo aplicador de corretivos, um mecanismo para aplicação de adubo com opção de variação da profundidade de aplicação $(0,40$ e $0,80 \mathrm{~m})$, uma enxada rotativa para destorroamento do solo e um sistema para realização da canteirização (formação de canteiros em faixas) (Figura $1)$.

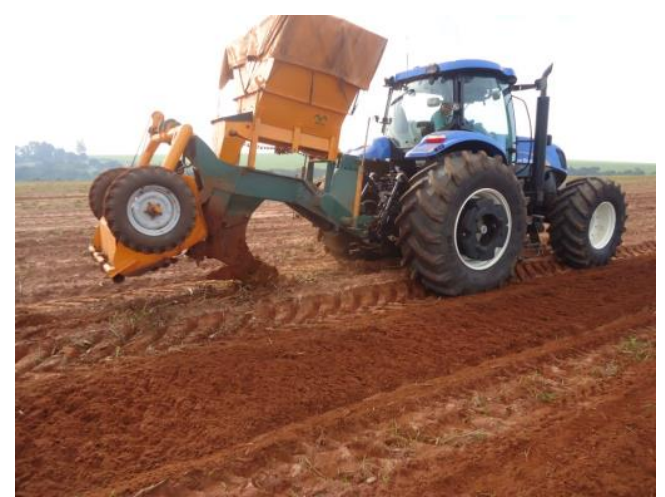

Figura 1. Equipamento utilizado para preparo profundo e canteirizado do solo.

O equipamento com medições de $3,00 \mathrm{~m}$ de altura total, largura total de 3,70 m e largura de trabalho de 1,20 $\mathrm{m}$; o solo da área experimental classificado como Argissolo Vermelho-Amarelo, textura arenosa média (EMBRAPA, 2013).

A pesquisa foi realizada em dois blocos, manejo do solo com o preparo profundo (PP); 1 metro de mobilização e outro com o preparo convencional (PC); 0,45 metros de mobilização do solo, divididos em 4 faixas, com 4 repetições de transeptos de 15 pontos, totalizando 16 transeptos para cada tipo de preparo. $\mathrm{O}$ preparo do solo constituiu-se da operação de uma gradagem e uma subsolagem.

A variável resistência à penetração do solo (RPS) foi avaliada em forma de transepto. Para a amostragem foram coletados 15 pontos, sendo 4 pontos na área de tráfego de cada lado do canteiro e 7 pontos no canteiro (Figura 2).

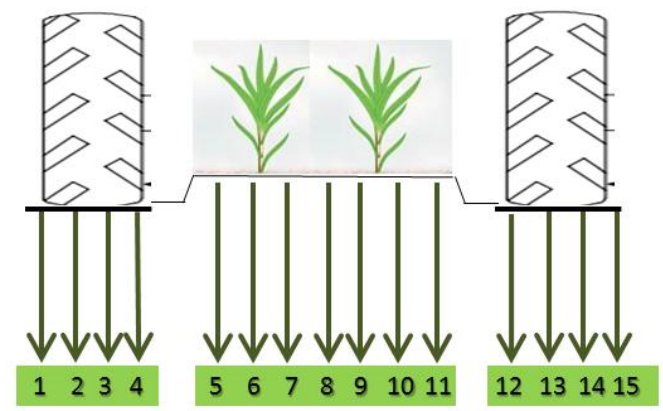

Figura 2. Croqui de amostragem dos pontos para a construção do transepto. 
Para a confecção dos transeptos, o experimento foi dividido em 3 seções com amostragens de 16 transeptos com 15 pontos amostrais em cada um e em cada bloco (PP e PC), apresentando um total geral de 480 (240 mais 240) pontos coletados.

O plantio, em ambos os tratamentos, foi realizado com espaçamento entre fileiras das plantas no canteiro de $0,90 \mathrm{~m}$ por $1,50 \mathrm{~m}$ e entre centro de linha de tráfego de 2,40 m.

Para confecção dos mapas dos transeptos para os tratamentos foi utilizado o software Gamma Design (GS+) (Robertson, 2000), iniciando utilizando a planilha para organização dos dados coletados, variograma, krigagem e por último o índice de dependência espacial (IDE).

Para análise do índice de dependência espacial (IDE), foram utilizados a relação $\mathrm{C} 0 /(\mathrm{C} 0+\mathrm{C})$ e os intervalos propostos por Cambardela et al. (1994), que consideraram a dependência espacial fraca (IDE > 75\%), moderada $(25 \%<$ IDE < $75 \%)$ e forte (IDE < 25\%).

Para a obtenção da resistência à penetração do solo (RPS) foi utilizado a Unidade Móvel de Amostragem do Solo - UMAS (Figura 3), desenvolvida pelo NEMPA, Núcleo de Ensaios de Máquinas e Pneus Agrícolas do Departamento de Engenheira Rural, da FCA/UNESP, Campus de Botucatu/SP, por Lanças e Santos (1998).

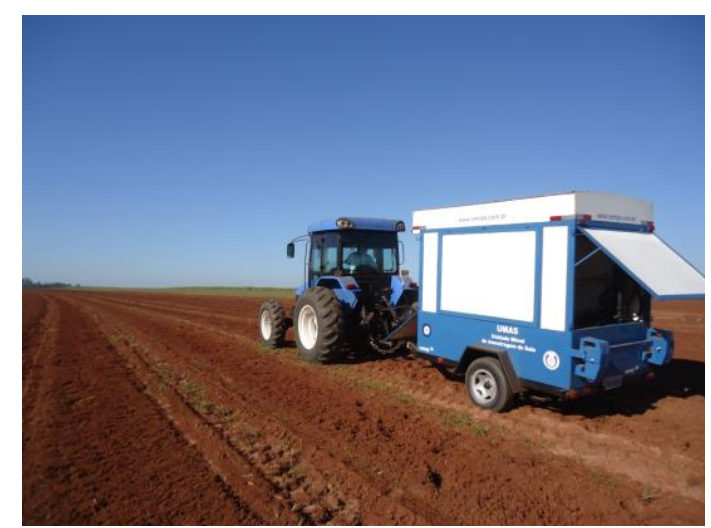

Figura 3. Avaliação após o preparo do solo com a Unidade Móvel de Amostragem do solo (UMAS).

Por meio dos valores de resistência à penetração do solo calculou-se o Índice de Cone (IC), conforme descrito pelos autores anteriormente citados. Para a avaliação dos pontos de resistência à penetração do solo foi utilizado o penetrômetro (Figura 4) instalado na parte de trás da Unidade Móvel (UMAS).

Para as coletas de dados utilizou-se uma célula de carga modelo "CS 1000" da marca "Líder" encaixada na haste, sendo o deslocamento da haste registrado por um potenciômetro multivoltas. A velocidade do penetrômetro foi estabelecida em $30 \mathrm{~mm} \mathrm{~s}^{-1}$ conforme norma da ASABE (2012).

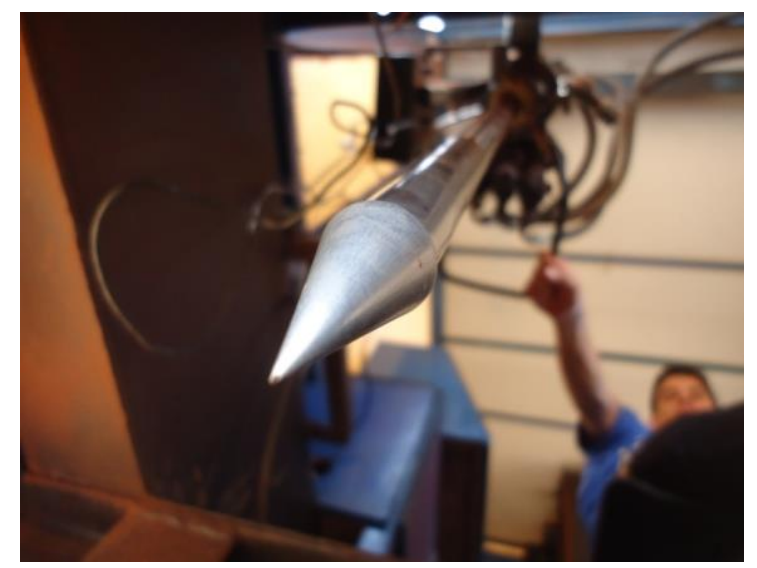

Figura 4. Amostragem de resistência nas coletas de dados.

Para a amostragem de preparo do solo realizou-se avaliações no PP e no PC, nas quais foram coletados quinze pontos para a construção do transepto, iniciando a coleta na metade da linha de tráfego, passando pelas linhas da cultura até a outra linha de tráfego.

A UMAS possui um notebook com um software criado em "Labview" da "National Instruments". O hardware utilizado foi o dispositivo de aquisição de dados "NIDAQ 6009", também da "National Instruments" que se comunica com um computador portátil via USB gerando o gráfico de resistência à penetração do solo para cada ponto.

A resistência à penetração do solo foi obtida até a profundidade de $0,45 \mathrm{~m}$, sendo as avaliações divididas posteriormente em camadas de 0 a $0,15,0,15$ a 0,30 e 0,30 a $0,45 \mathrm{~m}$.

\section{Resultados e Discussão}

$\mathrm{Na}$ Tabela 1 são apresentados os valores dos índices de geoestátistica e suas varioagramas conforme as avaliações propostas entre os manejos.

Quanto ao índice de dependência espacial, as camadas avaliadas apresentaram forte dependência espacial de acordo com Cambardella et al. (1994).

Lima et al. (2009) trabalhando a densidade do solo como indicador de compactação na resistência do solo à penetração em vegetação secundária em regeneração natural também encontrou dependência espacial forte.

Nas figuras 5 PP e 6 PC estão representadas as dispersões dos pontos divididos em grupos pelo programa e atribuindo um formato para cada grupo

Os maiores valores de resistência do solo foram nas zonas de tráfego, para Balastreire (1987) onde se verifica maior ocorrência de camadas compactadas não somente nos locais de deslocamento como também nas áreas adjacentes, visto que o efeito da pressão ocorre em forma de bulbos no local de aplicação da carga. 
Tabela 1. Semivariograma e parâmetros de ajuste para os diferentes preparos e camadas

\begin{tabular}{cccccccc}
\hline Preparo do solo & Camadas $(\mathrm{m})$ & Modelo & $\mathrm{A}(\mathrm{m})$ & $\mathrm{Co}$ & $\mathrm{C}_{0}+\mathrm{C}$ & $\mathrm{IDE}(\%)$ & $\mathrm{R}^{2}(\%)$ \\
\hline PP & $0-0,15$ & Gaussiano & 0,46 & 0,001 & 0,38 & 0.003 & 95 \\
PP & $0,15-0,30$ & Gaussiano & 0,46 & 0,059 & 2,67 & 0.022 & 92 \\
PP & $0,30-0,45$ & Gaussiano & 0,48 & 0,03 & 3,8 & 0.008 & 93 \\
**PC & $0-0,15$ & Gaussiano & 0,60 & 0,042 & 0,19 & 0.221 & 82 \\
PC & $0,15-0,30$ & Gaussiano & 0,38 & 0,001 & 0,79 & 0.001 & 98 \\
PC & $0,30-0,45$ & Efeito Pepita Puro & - & - & - & - & -
\end{tabular}

*Preparo Profundo (PP); ** Preparo Convencional (PC). Co: efeito pepita; C0+C: patamar; R2: coeficiente de correlação; IDE: índice de dependência espacial.

Na figura 7, observa-se que o valor do coeficiente de regressão pela validação cruzada no preparo PP teve maior valor; 97\% dos dados são representados pelas regressões presentes e $89 \%$ para PC. As figuras foram obtidas diretamente do programa em uso que apresenta somente a versão em inglês.

A tendência dos dados se ajustaram aos mesmos modelos, variando o alcance e patamar entre os manejos (Figura 8).

Oliveira et al. (2014) estudando semivariograma escalonado no planejamento amostral da resistência à penetração e umidade de solo com cana-de-açúcar encontrou modelos diferentes dos encontrados nesse trabalho, esférico e exponencial para esta mesma variável. Teores de argila, silte e areia ou a formação morfológica podem influenciar nas variações de valores de compactação do solo.
Na figura 9 e 10 estão representados os mapas de resistência à penetração do solo para as camadas de 015, 0,15- 0,30 e 0, 30 -0,45 m para o preparo PP e PC.

Os gráficos evidenciam a diferença entre a compactação das zonas de tráfego em relação ao canteiro para os dois preparos do solo e as camadas avaliadas. O PP mostra uma área maior na cor verde que representa os menores valores de compactação do solo e a homogeneidade do preparo em relação ao PC. Trabalhando com mapas de isolinhas, Cortez et al. (2014), utilizando-se da interpolação por krigagem para a representação da RP em perfil do solo, após deslocamento dos tratores para os diferentes números de passadas verificaram altos valores de RP em perfil; superiores a 6,0 $\mathrm{MPa}$, o que é demonstrado pela distribuição de zonas compactadas a partir da camada 0,10-0,20 $\mathrm{m}$ até a camada 0,30-0,40 m.
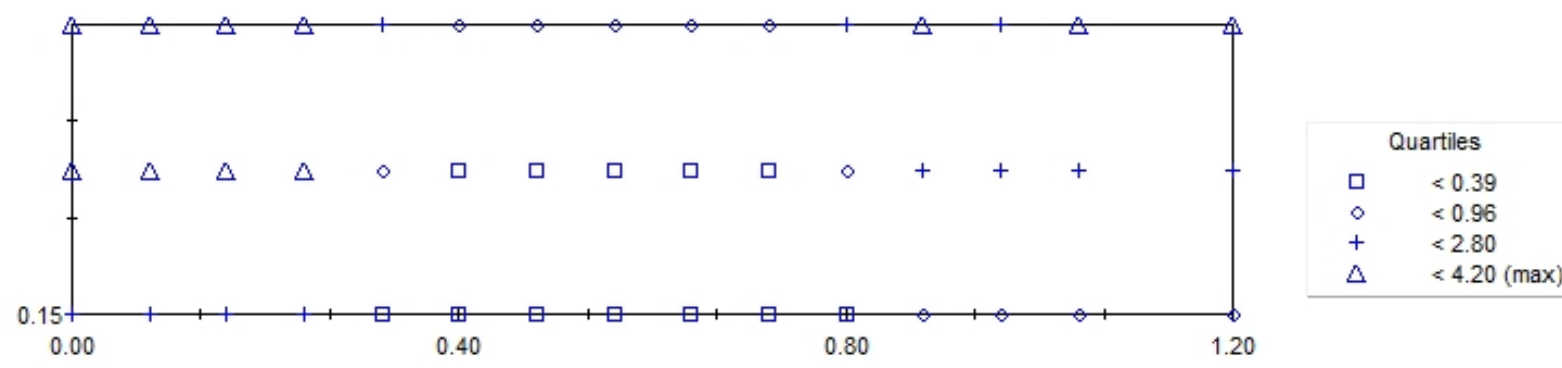

Figura 5. Dispersão dos pontos conforme os valores de compactação para preparo profundo (PP) para o transepto de 1,20 m.
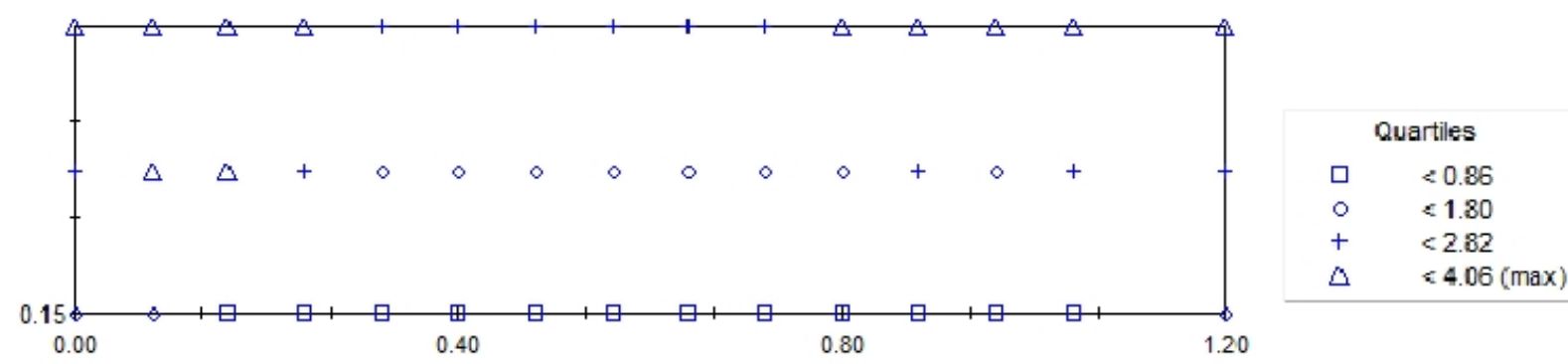

Figura 6. Dispersão dos pontos conforme os valores de compactação para preparo convencional (PC) para o transepto de 1,20 m. 
A

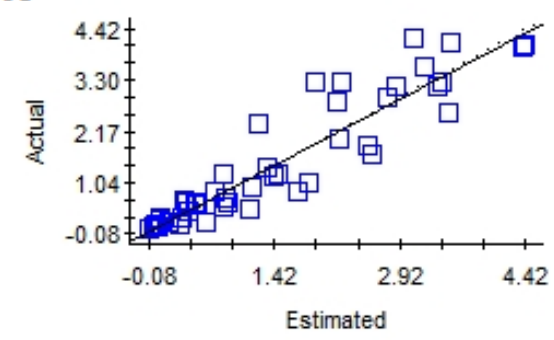

Regression coefficient $=0.970(\mathrm{SE}=0.060, \mathrm{r} 2=0.859$ $\mathrm{y}$ intercept $=0.06$, SE

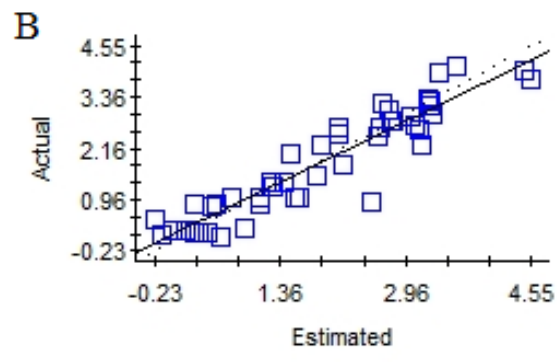

Regression coefficient $=0.898(\mathrm{SE}=0.053, \mathrm{r} 2=0.868$, $\mathrm{y}$ intercept $=0.14$, SE Prediction $=0.448$ )

Figura 7. Validação cruzada do preparo profundo - PP (a) e preparo convencional - PC (b).

A

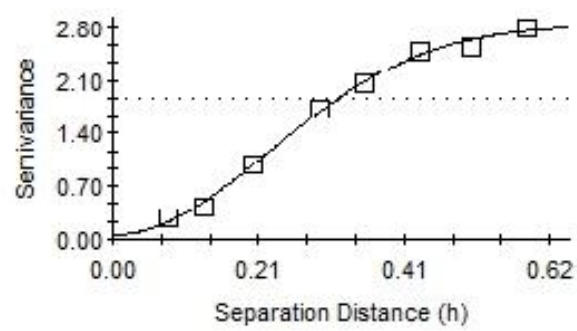

Gausslan model $(C O=0.05900 ; C O+C=2.83800 ; A O=0.31 ;$ RSS $=0.0204$
B Isotropic Variogram

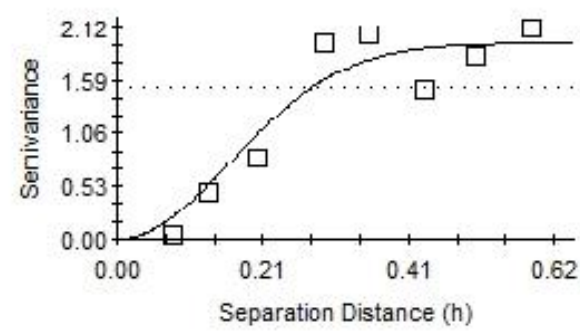

Gausslan model $\left(\mathrm{CO}=0.00100 ; \mathrm{CO}_{0}+\mathrm{C}=1.96600 ; \mathrm{AO}=0.23\right.$; RSS $=0.499$ )

Figura 8. Tendência dos dados para preparo profundo - PP (a) e preparo convencional - PC (b).

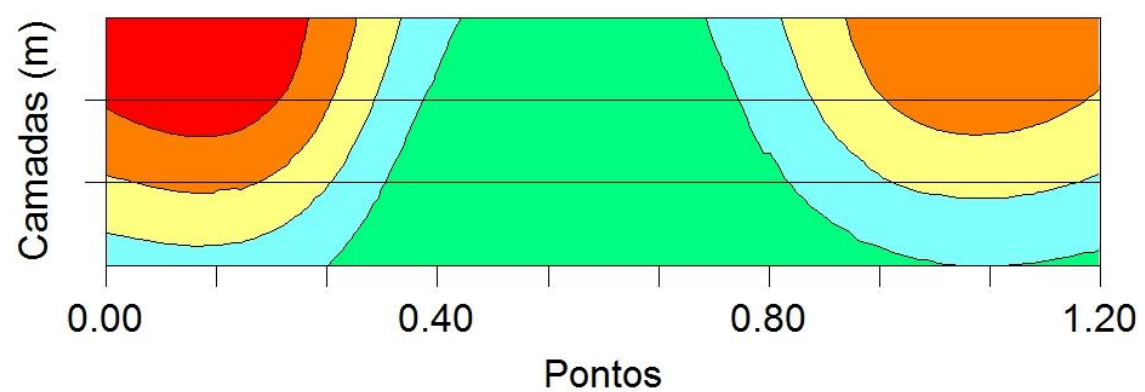

$\mathrm{C}(\mathrm{MPa})$

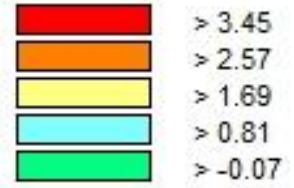

Figura 9. Mapa representando o transepto do PP em metros.

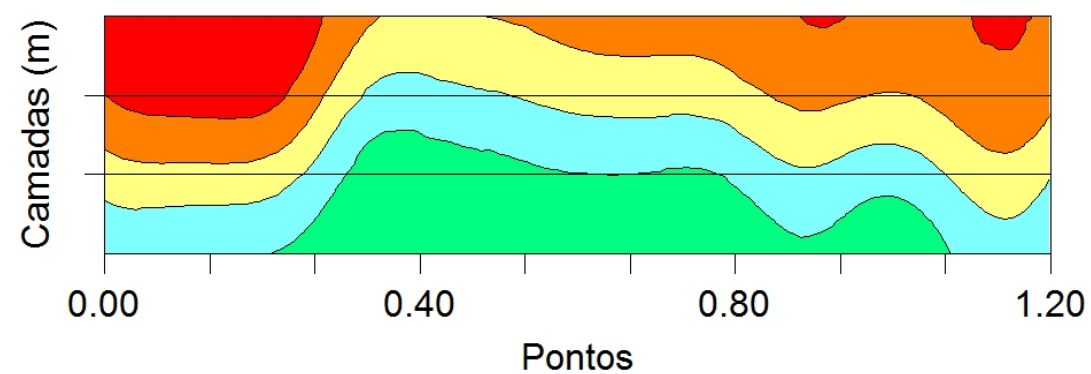

IC $(\mathrm{MPa})$

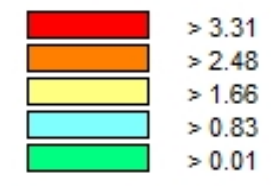

Figura 10. Mapa representando o transepto PC em metros. 
Apesar de se usar o piloto assistido no PC o manejo teve zonas de maior compactação. Para Souza et al., (2014) sistemas de gestão com o controle de tráfego baseado no ajuste da largura da pista de "Tractortrailer set" e a utilização de um piloto assistido preservando a qualidade física do solo nas linhas de plantas resultou em maior compactação sob o centro da entrelinha.

As fotos 5, 6, 7, 8, 9 e 10 utilizadas neste trabalho são fiéis às geradas pelo software Gamma Desing.

\section{Conclusões}

Os dados demonstraram que a camada de 0,30 a 0,45 $\mathrm{m}$ no preparo convencional foi o único que não apresentou dependência espacial.

$O$ uso da geoestatística como ferramenta na agricultura teve resposta satisfatória e o programa GS + mostrou-se confiável na interpolação dos mapas para a variável de compactação do solo.

\section{Agradecimentos}

Ao Núcleo de Ensaio de Máquinas e Pneus Agroflorestais (NEMPA) pela oportunidade de desenvolvimento deste trabalho.

Às Faculdades Integradas de Bauru pela realização dessa graduação.

\section{Referências Bibliográficas}

ASABE. AMERICAN SOCIETY OF AGRICULTURAL AND BIOLOGICAL ENGINEERS. Soil cone penetrometer. Saint Joseph, 2006. p. 902-904.

BALASTREIRE, L. A. Máquinas Agrícolas. São Paulo-SP: Manole, 1987. 310 p.

CAMBARDELLA, C. A.; MOORMAN, T. B.; NOVAK, J. M.; PARKIN, T. B.; KARLEN, D. L.; TURCO, R. F.; KONOPKA, A. E. Field-scale variability of soil properties in central Iowa soils. Soil Science Society of America Journal, Madison, v. 58, p. 1501-1511, 1994.

CARRARA, M.; CASTRIGNANÒ, A.; COMPARETTI, A.; FEBO, P.; ORLANDO, S. Mapping of penetrometer resistance in relation to tractor traffic using multivariate geostatistics. Geoderma, v.142, p. 294-307, 2007.

CONAB. COMPANHIA NACIONAL DE ABASTECIMENTO. Acompanhamento da safra brasileira de cana-de-açúcar, v. 2 - Safra 2015/16, n. 4 - Quarto levantamento, Brasília-DF, 2016. p. 1-76.

CORTEZ, J. W.; OLSZEVSKI, N.; PIMENTA, W. A.; PATROCÍNIO FILHO, A. P.; SOUZA, E. B.; NAGAHAMA, H. J. Avaliação da intensidade de tráfego de tratores em alguns atributos físicos de um Argissolo amarelo. Revista Brasileira de Ciência do Solo, Viçosa-MG, v. 38, n. 3, p.1000-1010, 2014.
EMBRAPA. EMPRESA BRASILEIRA DE PESQUISA AGOPECUÁRIA. Sistema Brasileiro de Classificação de Solos. Centro Nacional de Pesquisa de Solos. 6. ed. Rio de Janeiro-RJ, 2013. 412 p.

LANÇAS, K. P.; SANTOS FILHO, C. A. Penetrômetro hidráulico-eletrônico equipado com DGPS para avaliação da compactação do solo. La Plata: Editorial de la U.N.L.P, 1998. p. $570-576$.

LANDIM, P. M. B. Sobre geoestatística e mapas. CampinasSP: Editora Terræ Didatica, 2006. p.19-33.

LIMA, J. S. S.; SATTLER, M. A.; PASSOS, R. R.; OLIVEIRA, P. C.; SOUZA, G. S. Variabilidade espacial de atributos físicos de um Argissolo vermelho-amarelo sob pastagem e vegetação secundária em regeneração natural. Revista Engenharia Agrícola, Jaboticabal-SP, v. 29, n. 2, p.185-195, 2009.

OLIVEIRA, I. A.; CAMPOS, M. C. A.; AQUINO, R. E.; MARQUES JUNIOR, J.; FREITAS, L.; SOUZA, Z. M. Semivariograma escalonado no planejamento amostral da resistência à penetração e umidade de solo com cana-deaçúcar. Revista Ciências Agrárias, Recife-PE, v. 57, n. 3, p. 287-296, 2014.

PACHECO, E. P.; CANTALICE, J. R. B. Compressibilidade, resistência à penetração e intervalo hídrico ótimo de um Argissolo Amarelo cultivado com cana-de-açúcar nos Tabuleiros Costeiros de Alagoas. Revista Brasileira de Ciência do Solo, Viçosa-MG, v. 35, n. 2; p. 403-415, 2011.

PAULINO, A. F.; MEDINA, C. C.; AZEVEDO, C. B.; SILVEIRA, K. R. P.; TREVISAN, A. A.; MURATA, I. M. Escarificação de um Latossolo Vermelho na pós-colheita de soqueira de cana-de-açúcar. Revista Brasileira de Ciência do Solo, Viçosa-MG, v. 28, n. 5; p.911-917, 2004.

ROBERTSON, G. P. GS+: Geostatistics for theenvironmental sciences GS+ User's Guide. Plainwell: Gamma Desing Software, 2000. 152 p.

ROQUE, A. A. O.; SOUZA, Z. M.; ARAÚJO, F. S.; SILVA. G. R. V. Atributos físicos do solo e intervalo hídrico do solo de um Latossolo Vermelho distrófico sob controle de tráfego agrícola. Ciência Rural, Santa Maria-RS, v. 41, n. 9, p. 15361542,2011

SEGATO, S. V.; PINTO, A. S.; JENDIROBA, E.; NÓBREGA, J. C. M. Atualização em produção em cana-deaçúcar. Piracicaba-SP: Livroceres, 2006. 415p.

SMITH, R.; ELLIES, A.; HORN, R. Modified Boussinesq_s equations for nonuniform tire loading. Journal of Terramechanics, Grã Bretanha, v. 37, p. 207-222, 2000.

SOEHNE, W. Fundamental of pressur distribution and soil compactation under tractor tires. Agricultural Engineering, Michigan, v. 39, n. 5, p. 276-281, 1958.

SOUZA,G. S.; SOUZA, Z. M.; SILVA, R. B.; BARBOSA, R. S.; ARAÚJO, F. S. Effects of traffic control on the soil physical quality and the cultivation of sugarcane. Revista Brasileira de Ciência do Solo, Viçosa-MG, v. 38, n.2; p.135146, 2014. 
TRANGMAR, B. B.; YOST, R. S.; UEHARA G. Application of geostatistics to spatial studies of soil properties. Advances in Agronomy, Newark-USA, v.38, p.45-94, 1985.

VASCONCELOS, R. F. B.; CANTALICE, J. R. B.; MOURA, G. B. A.; RLIM, M. M.; MONTENEGRO, C. E. V. Compressibilidade de um Latossolo Amarelo distrocoeso não saturado sob diferentes sistemas de manejo da cana-de-açúcar. Revista Brasileira de Ciência do Solo,Viçosa-MG, v.36, n.2; p.525-536, 2012.
WALTER, A.; DOLZAN, P.; QUILODRÁN, O.; GARCIA, J.; SILVA, C.; PIACENTE, F.; SEGERSTEDT, A. A sustainability analysis of the Brazilian ethanol. CampinasSP: Unicamp, 2008. 167 p. 